\title{
AVALIAÇÃO EXPERIMENTAL DA EFICIÊNCIA NA RETENÇÃO DE SEDIMENTOS SÓLIDOS, EM UM RESERVATÓRIO SEPARADOR DE PRIMEIRAS ÁGUAS DA CHUVA
}

\section{EXPERIMENTAL ASSESSMENT OF SOLID SEDIMENT RETENTION EFFICIENCY IN A FIRST FLOW SEPARATIONTANK}

\author{
Lineker Max Goulart Coelho \\ Centro Federal de Educação Tecnológica de Minas Gerais, CEFET-MG. (lineker.goulart@gmail.com) \\ Gabriela Cristina Marcelino \\ Engenheira civil, Instituto Federal de Minas Gerais, Santa Luzia - MG. (gabic.mar@gmail.com)
}

Leandro Alves Evangelista

Engenheiro civil, Instituto Federal de Minas Gerais, Santa Luzia - MG. (leandroalves1989@gmail.com)

Emily Louise Silva Amaral

Técnica em edificações, Instituto Federal de Minas Gerais, Santa Luzia - MG. (emilylouise.07.09.00@gmail.com)

Vinicius José de Aguiar Gomes Costa

Técnico em edificações, Instituto Federal de Minas Gerais, Santa Luzia - MG. (viniciuscostazero@hotmail.com)

Submissão: 30 de Abril de 2019

Aceite: 28 de Outubro de 2019

\section{Resumo}

O objetivo deste trabalho foi realizar uma avaliação experimental de um reservatório de separação de primeiras águas (RSPA) para aproveitamento de água de chuva. Inicialmente, dimensionou-se de forma regionalizada o volume do RSPA, concebido a partir de uma vazão dada pela chuva de projeto, calculada pelo método racional. Em seguida, elaborou-se uma geometria para o RSPA, com o objetivo de avaliar de forma experimental a sua eficiência na retenção de material particulado (sedimentos). O RSPA foi fabricado com tubos e conexões de policloreto de vinila (PVC) em escala real e foi instalado em um sistema de alimentação em uma edificação de ensino. Para essa avaliação, produziram-se sedimentos a partir de um processo de peneiramento, utilizando-se peneiras com aberturas padronizadas. O experimento consistiu em aplicar no RSPA uma vazão correspondente à chuva de projeto com uma concentração de sedimentos, simulando as condições das primeiras águas de chuva. Durante o experimento, realizaram-se amostragens na saída do RSPA e avaliou-se sua eficiência na retenção dos sedimentos inseridos na massa líquida. Os resultados indicam que a geometria escolhida do RSPA foi eficiente do ponto de vista experimental para a retenção de sólidos, obtendo uma remoção de cerca de $70 \%$ do material particulado.

Palavras-Chave: Reservatório separador de primeiras águas; aproveitamento de água da chuva; avaliação experimental.

\section{Abstract}

The aim of this paper was carry out an experimental assessment of a first flush tank (FFT) for rainwater harvesting. Firstly, the volume of the FFT was determined in a regionalized basis from a flow rate given by the design rain, calculated according to the rational method. Subsequently a geometry for the FFT was designed, with the objective of evaluating its efficiency in the retention of solid particulate material (sediments). The FFT was manufactured using pipes and connections of polyvinyl chloride (PVC) in full scale and was installed in a feed system located in a school building. The experiment consisted in apply to the FFT a flow correspondent to a design rain with a solids concentration simulating the conditions of the first water of a rain. During the experiment, sampling was performed at the outlet of the FFT, and its efficiency in the retention of the inserted sediments was evaluated. From the results it is noted that the FFT geometry tested was efficient in terms of experimental solid retention, achieving a removal rate of $70 \%$ of particulate material.

Keywords: First flow separation tank; rainwater harvesting; experimental assessment. 


\section{INTRODUÇÃO E FUNDAMENTAÇÃO TEÓRICA}

O uso de água de chuva para fins potáveis requer cuidados especiais que, muitas vezes, não são respeitados. Isso porque, para a água estar adequada para fins potáveis, ela deve apresentar algumas características físicas, químicas e microbiológicas que garantem que seu uso não provocará problemas de saúde aos indivíduos. Sendo assim, para considerar a água como potável, é necessário que ela passe por tratamentos que buscam remover ou reduzir a concentração de poluentes até que sejam atendidos os limites estabelecidos pela legislação.

Entretanto, devido à precariedade e escassez de recursos enfrentados pelas comunidades do semiárido, tais processos não são adotados, o que gera riscos à saúde e bemestar dessas populações. Sendo assim, o desenvolvimento de métodos simplificados de tratamento que possam ser incorporados ao modelo das cisternas do semiárido, tais como o desenvolvimento de sistemas de separação das primeiras águas, poderia proporcionar uma melhor qualidade de água utilizada pelas populações em questão, melhorando a qualidade de vida destas e minimizando riscos à saúde por ingestão da água de chuva.

Dentre os elementos que compõem um sistema de aproveitamento de água de chuva, o reservatório de separação das primeiras águas (RSPA) ou reservatório de autolimpeza representa um importante elemento para a garantia da qualidade da água, pois este evita que as águas escoadas inicialmente, as quais apresentam elevada carga sólida, sejam enviadas ao reservatório principal de armazenamento de água de chuva (TOMAZ, 2012). Esse elemento tem por função reter as águas do início da chuva que, pelo fato de lavarem o telhado, carregam a poeira e demais resíduos nele depositado, apresentando, por isso, uma elevada concentração de poluentes e sólidos. Dessa forma, normalmente, descarta-se esta "primeira água de chuva" direcionando-a para um reservatório específico, denominado reservatório das primeiras águas. De acordo com Novak, Giesen e Debusk. (2014), obtém-se uma melhoria significativa da qualidade da água de chuva apenas desprezando a primeira descarga de um evento pluviométrico. Entretanto, existem poucos trabalhos científicos dedicados ao estudo do reservatório de primeiras águas (OHNUMA et al.,
2014)

Atualmente, a norma vigente no Brasil que prescreve diretrizes para o aproveitamento de água da chuva em áreas urbanas para fins não potáveis é a norma regulamentadora da Associação Brasileira de Normas Técnicas (ABNT) NBR-15527:2007. Esta norma prescreve a implantação das partes de um sistema para aproveitamento de água da chuva, sem considerar os efeitos decorrentes das diversas regiões e climas do país. Em especial, a norma prescreve que quando for implantado um dispositivo para descarte da água inicial, ou reservatório de separação das primeiras águas (RSPA), sem o dimensionamento de um projetista, recomenda-se o descarte de $2 \mathrm{~mm}$ da precipitação inicial. Como esse item prescrito não leva em consideração quaisquer parâmetros relativos às características regionais ou configurações geométricas do RSPA utilizado, a sua adoção pode conceber um descarte de água da chuva elevado ou aquém do necessário.

Diferentemente do prescrito na NBR 15527:2007 (ABNT, 2007), Melo e Andrade Neto (2007) realizaram estudos experimentais quanto à qualidade da água oriunda do RSPA e concluíram que 0 descarte do primeiro $\mathrm{mm}$ de água, geralmente, já é suficiente para a remoção da maior parte dos poluentes das primeiras águas de chuva captadas. Existem ainda métodos de determinação do volume do RSPA que consideram as características do sistema para determinação do volume ideal, tais como os procedimentos descritos em Martinson e Thomas (2009) e Mierzwa et al. (2007).

Salienta-se ainda que a NBR 15527:2007 expõe apenas um critério simplificado para cálculo do volume do RSPA, mas não apresenta diretriz quanto à geometria de tal elemento. Esta, por sua vez, pode afetar diretamente a eficiência de remoção de poluentes, tendo em vista que a forma do RSPA pode favorecer ou não a retenção de sólidos oriundos das primeiras chuvas. Os efeitos da geometria do RSPA na qualidade da água enviada ao reservatório principal são discutidos em Andrade Neto (2013).

Dessa forma, investigações relacionadas com o formato do RSPA podem resultar em melhor eficiência de remoção de poluentes das águas a serem encaminhadas para os sistemas de aproveitamento de água de chuva, proporcionando uma melhor qualidade da água que é direcionada para o reservatório principal.

Do ponto de vista científico, de acordo com 
Tomaz (2012), Novak, Giesen e Debusk (2014) e Medeiros (2016), o desenvolvimento e aperfeiçoamento de cada um dos elementos do sistema de aproveitamento são cruciais para a sua popularização, pois a busca por alternativas de alta eficiência com baixo custo é um fator essencial para a disseminação de tal prática e um nicho de projeto para novas pesquisas científicas. Além disso, para Andrade Neto (2013), o aperfeiçoamento dos dispositivos de descarte das primeiras águas possui papel fundamental para melhoria das características sanitárias da água de cisternas de aproveitamento de água de chuva. Recentemente, Notaro, Liuzzo e Freni (2016) evidenciaram que o método utilizado na determinação do volume do reservatório de armazenamento é crucial para garantir a confiabilidade de atendimento do sistema de consumo de água de chuva. Bitterman et al. (2016), por sua vez, desenvolveram indicadores de desempenho para o controle mais efetivo dos sistemas de aproveitamento de água de chuva.

Ressalta-se que a própria estrutura da edificação e os materiais utilizados podem influenciar na qualidade da água de chuva captada. Cardoso (2009) investigou o efeito dos tipos de telha na qualidade da água de chuva captada e observou diferenças entre telhados que utilizam telha cerâmica e telha metálica. Já Piccoli (2015) forneceu uma importante contribuição para o cenário brasileiro, uma vez que estudou as características físico-químicas das águas de chuva apresentando valores médios de referência para cada parâmetro avaliado, aspecto importante no projeto de sistemas de aproveitamento de água de chuva.

Especificamente com relação aos reservatórios de primeiras águas, Ohnuma et al. (2014) mostraram a eficiência de remoção de turbidez ao se utilizar tal dispositivo em um sistema de aproveitamento, alcançando valores de turbidez média de 64 NTU e de sólidos totais de $110 \mathrm{mg} / \mathrm{L}$. Ainda sobre reservatório das primeiras águas, Lima, Carvalho e Figueiras (2016) atestaram a eficiência deste elemento para redução dos coliformes na água. Novak, Giesen e Debusk (2014) relataram eficiências de remoção de sólidos de $80 \%$ em sistemas RSPA. Em Gardner, Baisden e Millar (2004), o estudo de retenção de sólidos com um RSPA alcançou $65 \%$ de eficiência. Já Su (2007) obteve remoções de sólidos variando entre $30 \%$ e $40 \%$.

Com esta perspectiva, o objetivo deste estudo é propor uma geometria para um RSPA e avaliar, experimentalmente, a sua eficiência de retenção de sólidos.

\section{MATERIAIS E MÉTODOS}

O estudo em questão foi realizado com base nas seguintes etapas, cujos procedimentos são:

- Determinação do volume do RSPA

- Definição da geometria do RSPA

- Construção do RSPA e do sistema de alimentação

- Preparação do material particulado e elaboração da curva de calibração

- Execução do experimento.

\section{Vazão correspondente à chuva de projeto $e$ volume do RSPA}

Para determinação da vazão correspondente à chuva de projeto, utilizou-se método racional, que estabelece uma associação da precipitação com a vazão produzida. A utilização do método racional é aplicada a áreas de captação menores que $3 \mathrm{~km}^{2}$, e consiste na aplicação da equação (1) (COELHO et al., 2018):

$$
\mathrm{Q}=\frac{\mathrm{CXIXA}}{360}
$$

Em que:

- Q é a vazão, em m ${ }^{3} / \mathrm{s}$

- C é a coeficiente de escoamento superficial, variável entre 0 e 1

- I é a intensidade média da chuva, em $\mathrm{mm} / \mathrm{h}$

- A é a área de captação, em m².

A intensidade média da chuva foi calculada utilizando-se a equação IDF, que correlaciona a intensidade, a duração e a frequência da chuva, corrigidas por parâmetros empíricos de cada região, dada pela equação (2).

$$
I=\frac{k \times T^{a}}{(t+b)^{\mathrm{C}}}
$$

Onde:

- $k$, a, b e c são parâmetros empíricos específicos de cada região

- Tr é o tempo de retorno da chuva, em anos

- t é a duração da chuva, em minutos

Para a determinação dos parâmetros empíricos k, a, b e 
c, empregou-se o software Pluvio 2.1, desenvolvido pela Universidade Federal de Viçosa, que determina os parâmetros da equação IDF para diversas cidades do Brasil. Nos cálculos, utilizaram-se os parâmetros referentes à cidade de Belo Horizonte - MG, encontrando-se os seguintes valores: $k=682,93, a=0,169, b=$ $3,994, \mathrm{c}=0,671$.

$\mathrm{Na}$ determinação da intensidade da chuva de projeto, utilizaram-se o tempo de retorno de 1 ano e duração da chuva de 25 minutos. Para a área de captação, adotou-se $80 \mathrm{~m}^{2}$, área comum em coberturas de residências de pequeno a médio porte.

Encontrando-se a vazão correspondente à chuva de projeto, calculou-se o tempo de enchimento do volume útil do RSPA, segundo a metodologia utilizada por Mierzwa et al. (2007), que propõe a utilização da equação (3). O objetivo deste trabalho não é descrever os procedimentos relacionados a esta abordagem; detalhes sobre esta metodologia são apresentados pelos seus desenvolvedores, Mierzwa et al. (2007).

$$
\mathrm{t}=\frac{2 \mathrm{~A}}{\mathrm{a}^{2}}\left[\mathrm{Qh}\left(\frac{\mathrm{Q}-\mathrm{a} \sqrt{\mathrm{h}}}{\mathrm{Q}-\mathrm{a} \sqrt{\mathrm{Li}}}\right)+\mathrm{a}(\sqrt{\mathrm{h}}-\sqrt{\mathrm{Li}})\right](3)
$$

Onde:

- t é o tempo para que se atinja o volume útil do RSPA, em segundos

- A é a área total de captação, em $\mathrm{m}^{2}$

- Q é a vazão de entrada do RSPA, em $\mathrm{m}^{3} / \mathrm{s}$

- h é a cota na qual a água atinge a saída do RSPA, em m

- L é o comprimento da tubulação de descarte, em $m$

- i é a inclinação da tubulação de descarte, em $\mathrm{m} / \mathrm{m}$

- a é o coeficiente de correção hidráulico, dado pela equação (4)

$$
\mathrm{a}=\frac{\pi \times \mathrm{D}^{2}}{4} \frac{1}{\sqrt{\mathrm{C}}} \sqrt{2 \mathrm{~g}}
$$

Em que:

- D é o diâmetro da tubulação de saída do RSPA

- g é a aceleração da gravidade

- C é o coeficiente relacionado aos fatores de atrito, determinado pela equação (5), onde $f$ é o fator de atrito de Darcy Weisbach de perda distribuída e k é coeficiente de perda de carga localizada, sendo iguais a 0,03 e 2, respectivamente

$$
\begin{aligned}
& \mathrm{C}=1+ \\
& \mathrm{f} \frac{\mathrm{L}}{\mathrm{D}}+\mathrm{K}
\end{aligned}
$$

Empregando-se as equações (3), (4) e (5), obteve-se o tempo necessário de enchimento do volume útil do RSPA. Com esse tempo, determina-se o seu volume, utilizando-se a equação (6).

$$
V=Q \cdot t
$$

\section{Em que:}

- $\quad V$ é o volume do RSPA, em $\mathrm{m}^{3}$

- $Q$ é a vazão de projeto, em $\mathrm{m}^{3} / \mathrm{s}$

- $t$ é o tempo de enchimento do RSPA, em segundos

\section{Definição da geometria do RSPA}

A partir do volume obtido, definiu-se uma configuração geométrica do RSPA que armazenasse o volume calculado e do sistema que seria utilizado para alimentar o RSPA e coletar a água oriunda deste. $\mathrm{O}$ procedimento de determinação do formato e das dimensões do RSPA é apresentado em Coelho et al. (2018). Neste estudo, definiu-se a geometria de um RSPA por meio de simulações hidráulicas computacionais, buscando otimizar a retenção de sólidos através da escolha do formato do RSPA.

Previamente, construiu-se o RSPA, um projeto virtual executado em Autocad $\AA$, de modo a permitir a visualização prévia do RSPA. A geometria do RSPA adotada é apresentada na Figura 1, e consiste em um reservatório cilíndrico com entrada e saída de água pela lateral em pontos diametralmente opostos e em alturas diferentes, havendo um desnível de $15 \mathrm{~cm}$ entre os eixos das tubulações de entrada e saída do RSPA. Essa geometria foi utilizada como base para a construção do RSPA em escala real, o qual foi empregado no estudo experimental.

\section{Construção do RSPA e do sistema de alimentação}

Para facilitar a disseminação do filtro projetado, buscou-se utilizar materiais de baixo 
custo e de fácil acesso no mercado. Sendo assim, os materiais selecionados para a construção do RSPA e do sistema de alimentação se limitaram ao uso de tubulações e conexões de PVC com diâmetros de $100 \mathrm{~mm}$ e $200 \mathrm{~mm}$.

\section{Figura 1: Forma geométrica escolhida para o RSPA: (a) vista lateral com dimensões em $\mathrm{cm}$ e (b) em perspectiva}

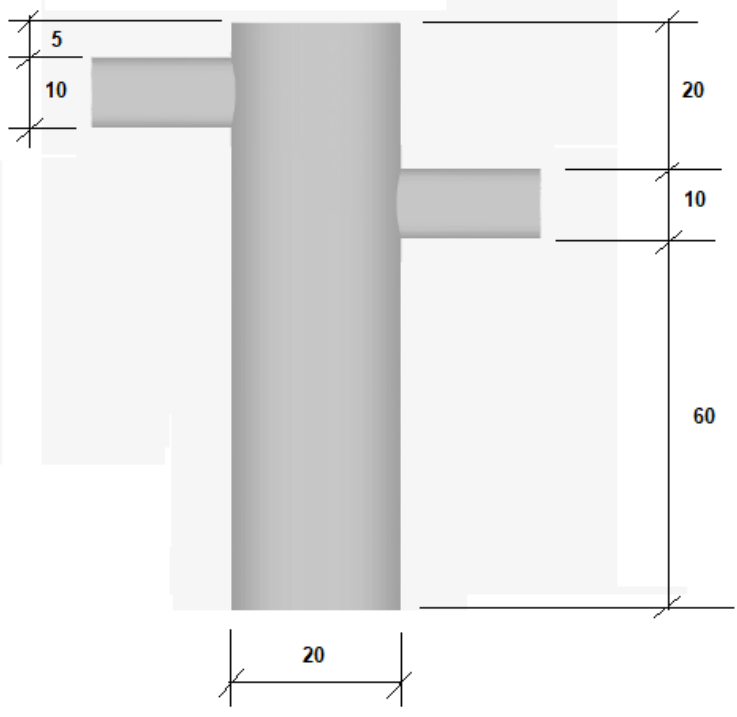

(a)

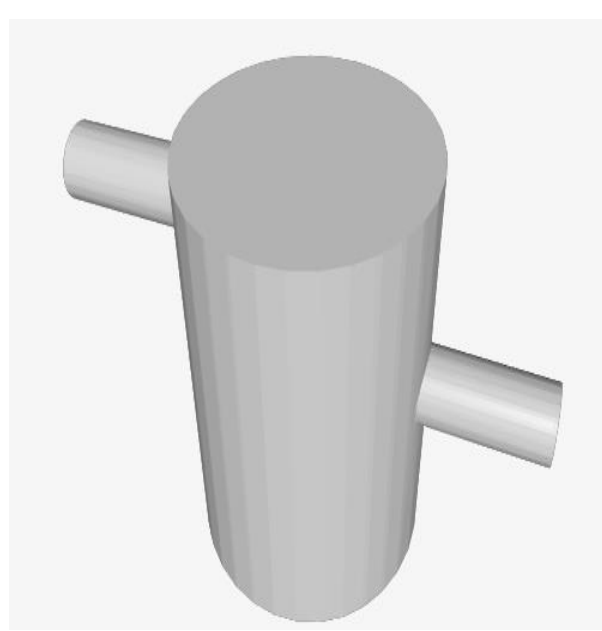

(b)

Fonte: Os autores.

A confecção das peças que compõem 0 RSPA projetado e o sistema de alimentação foi realizada com o auxílio de uma serra policorte e uma furadeira de bancada com broca do tipo serra copo, para execução dos cortes e furos necessários. Em seguida, as peças foram unidas e montadas no local de instalação do aparato experimental.

A instalação do sistema de alimentação do RSPA onde foram executados os experimentos efetuou-se na área externa do Instituto Federal de Minas Gerais - Campus Santa Luzia, localizado na cidade de Santa Luzia, Minas Gerais, situado na região metropolitana de Belo Horizonte.

Elaborou-se o projeto do RSPA e do sistema de alimentação utilizando-se os seguintes materiais: Tubo de PVC com DN100 para entrada e saída do RSPA; Tubo de PVC com DN200 para o corpo do RSPA; CAPs de PVC com DN200 para fechamento do topo e base do RSPA; Adesivo PVC e fita veda rosca para conexão da entrada e saída com o corpo do RSPA.

A Figura 2 apresenta detalhes das conexões utilizadas na montagem do RSPA. Nota-se, a partir dessa Figura, que o RSPA projetado apresenta duas partes principais que são conectadas por uma luva. A parte superior do RSPA possui a entrada e a saída da água, as quais se localizam em níveis distintos, estando a tubulação de entrada localizada $15 \mathrm{~cm}$ acima da tubulação de saída. A parte inferior do RSPA corresponde à porção principal de acumulação do reservatório, sendo formada por uma tubulação e um cap de DN200. A tubulação de entrada encontra-se a $5 \mathrm{~cm}$ do topo do RSPA e a tubulação de saída está a $20 \mathrm{~cm}$ do mesmo. Abaixo da tubulação de saída, o reservatório possui um comprimento de $60 \mathrm{~cm}$ em tubulação com DN 200,sendo esta a altura útil correspondente ao volume calculado.

\section{Preparação do material particulado e elaboração da curva de calibração}

Para realização do experimento, necessitouse obter material particulado utilizado como sedimento, observando que este deveria ter tamanho compatível com os materiais particulados que se encontravam depositados nas coberturas residenciais.

Segundo Ferreira (2016), material particulado pode ser definido como qualquer partícula sólida ou liquida presente na atmosfera com diâmetros inferiores a $100 \mu \mathrm{m}$. Convencionalmente, refere-se aos tamanhos das partículas utilizando-se diâmetros, mesmo que 
estas não possuam formatos esféricos.

Figura 2: Projeto do RSPA com detalhamento de peças utilizadas e cotas (dimensões em cm)

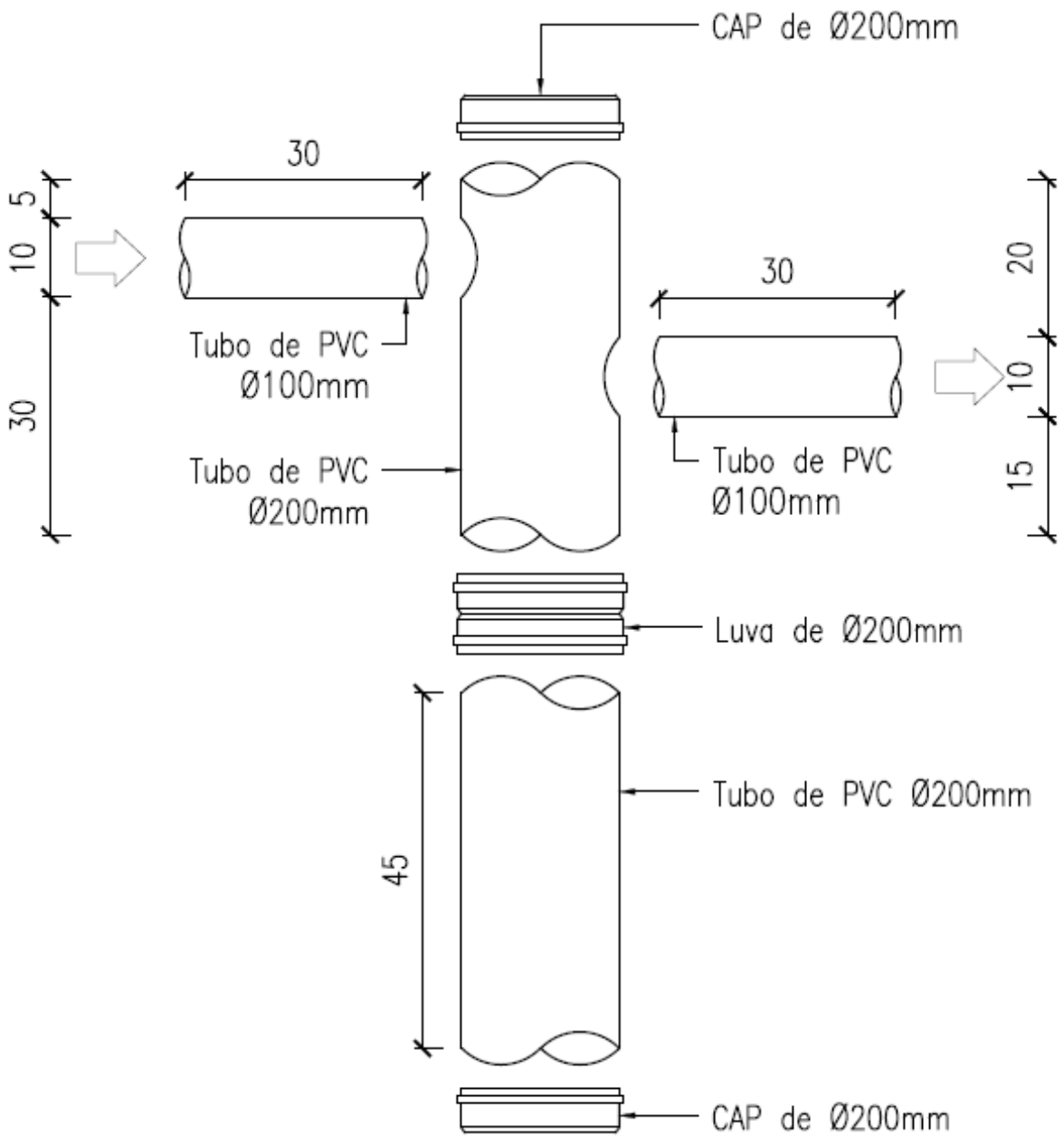

Fonte: Os autores.

A fim de utilizar um material particulado composto, este foi obtido a partir do peneiramento de dois solos distintos, um de cor bege com maior teor de areia e outro de cor avermelhado com maior teor de argila e silte. Realizou-se 0 peneiramento, passando-se ambos os solos individualmente por peneiras granulométricas padronizadas, com as seguintes aberturas, respectivamente: $2,36 \mathrm{~mm}, 1,00 \mathrm{~mm}, 0,850 \mathrm{~mm}$, $0,500 \mathrm{~mm}, 0,300 \mathrm{~mm}, 0,150 \mathrm{~mm}$ e $0,106 \mathrm{~mm}$. O material que passou pela peneira de $0,106 \mathrm{~mm}$ foi utilizado como sedimento para os experimentos e o restante foi descartado. Usou-se como sedimento uma composição de $50 \%$ do material peneirado do solo arenoso e $50 \%$ do material peneirado do solo silto-argiloso.

O material passante pela peneira com abertura de $0,106 \mathrm{~mm}$, na realização do peneiramento, resultou no sedimento utilizado no experimento, conforme apresentado na Figura 3.

Realizaram-se, ainda, registros fotográficos com aumento de 200 e 400 vezes dos sedimentos obtidos, utilizando-se um microscópio metalográfico, a fim de caracterizar o material visualmente quanto ao formato dos grãos, predominantemente, observados.

$\mathrm{Na}$ Figura 4, são apresentadas fotos dos sedimentos vistos a partir de um microscópio, sendo que as fotos (a), (b) e (c) estão com aumento de 200 vezes, e as fotos (d), (e) e (f) de 400 vezes. Analisando-se os registros fotográficos feitos do material utilizado como sedimento, Figura 4, observa-se que o sedimento obtido a partir do material silto-argiloso, Figura 4 (a) e (d), apresenta grãos com tamanhos mais constantes, com formato volumétrico, em que as três dimensões são aproximadamente iguais. No 
sedimento obtido a partir do solo arenoso, Figura 4 (b) e (e), observa-se maior variação dos tamanhos dos grãos do solo, com formatos lamelares, em que duas dimensões são muito maiores que a terceira. A Figura 4 (c) e 4 (e), por sua vez, apresentam a mistura dos dois sedimentos com uma composição de metade de cada tipo utilizado, notando-se, portanto, um aspecto dos grãos com características intermediárias devido à combinação dos dois sedimentos supracitados.

\section{Figura 3: Sedimentos misturados (50\% silto-argiloso e 50\% arenoso)}

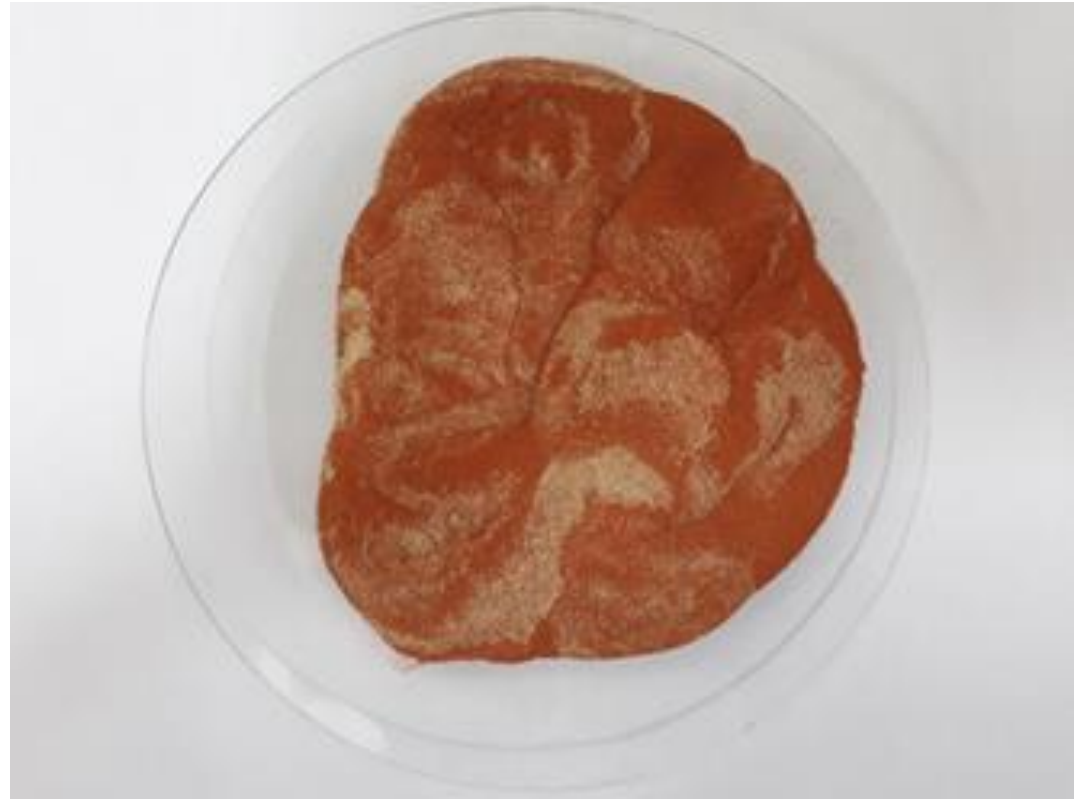

Fonte: Os autores.

Figura 4: Fotos dos materiais utilizados como sedimento observados em microscópio.
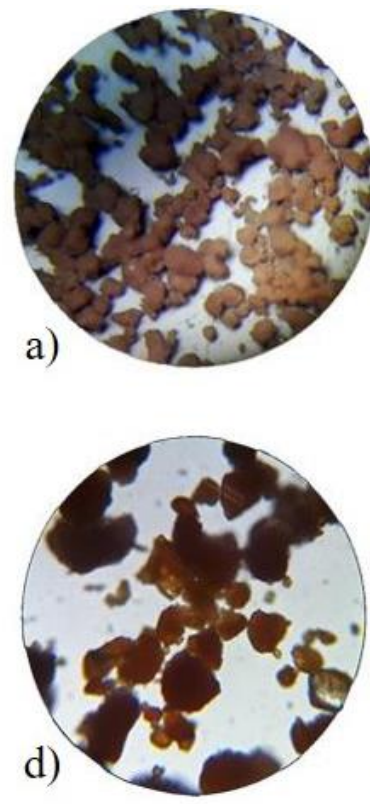

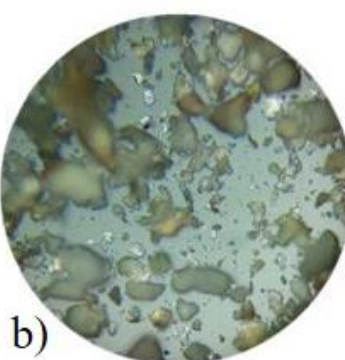

c)

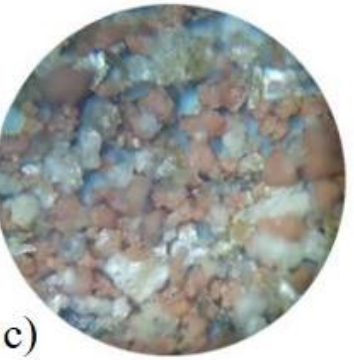

e)
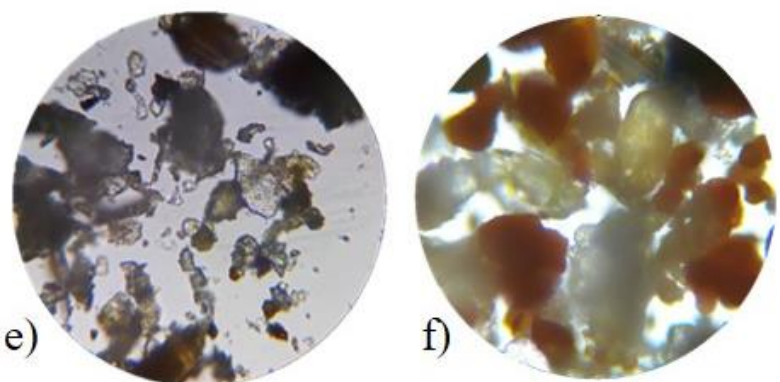

(a) material silto-argiloso aumentado 200 vezes, (b) material arenoso aumentado 200 vezes, (c) mistura dos dois materiais aumento de 200 vezes, (d) material silto-argiloso aumentado 400 vezes, (e) material arenoso aumentado 400 vezes e (f) mistura dos dois materiais aumentado 400 vezes Fonte: Os autores. 
Obtido o material particulado, procedeu-se com a construção da curva de calibração, utilizando-se o colorímetro $\mathrm{HI} 727$ da marca Hanna®. $O$ intuito da construção da curva de calibração é obter o valor de sólidos totais da amostra de maneira indireta, em função da sua cor aparente. Ressalta-se que o limite detecção do aparelho (500 PCU) é bem superior aos valores tipicamente encontrados em primeiras águas de chuva, sendo, portanto, adequada a utilização do aparelho em questão ao estudo. A curva foi elaborada de modo a abranger toda a faixa de capacidade de medição do aparelho. Para isso, fez-se uma mistura com concentração de $10 \mathrm{~g} / \mathrm{L}$ em um balão volumétrico de $1 \mathrm{~L}$ e realizou-se a medição do valor de cor aparente, que extrapolou o valor máximo de leitura do colorímetro (500 PCU). A concentração foi reduzida pela metade, ou seja, $5 \mathrm{~g} / \mathrm{l}$, e realizou-se novamente a medição da cor aparente. Em seguida, efetuaram-se diluições sucessivas e o valor da leitura do colorímetro para a respectiva concentração de sólidos totais. A redução da concentração de sólidos totais pela metade foi feita até que se encontrasse o valor de cor aparente igual à zero em duas leituras consecutivas, o que aconteceu na $10^{\underline{a}}$ e $11^{\text {a }}$ diluição, conforme apresentado na Tabela 1 . Então, com base nos dados de cor em função da concentração de sólidos totais de todo o intervalo de leitura do instrumento utilizado, construiu-se a curva de calibração mostrada na Figura 5 , a qual foi elaborada em escala logarítmica de base 10 . Os dados da curva foram usados para estimativa da concentração de sólidos totais das amostras coletadas no experimento com o RSPA.

\begin{tabular}{cc}
$\begin{array}{c}\text { Tabela 1: Determinação da cor aparente em função da } \\
\text { concentração de sólidos totais }\end{array}$ \\
\hline Concentração de sólidos totais (g/L) & Cor aparente (PCU) \\
\hline 10,00000 & 500 \\
5,00000 & 500 \\
2,50000 & 500 \\
1,25000 & 450 \\
0,62500 & 243 \\
0,31250 & 138 \\
0,15625 & 63 \\
0,07813 & 38 \\
0,03906 & 20 \\
0,01953 & 8 \\
0,00977 & 0 \\
0,00488 & 0
\end{tabular}

Fonte: Os autores.

Figura 5: Curva de calibração do colorímetro. Cor x Sólidos totais

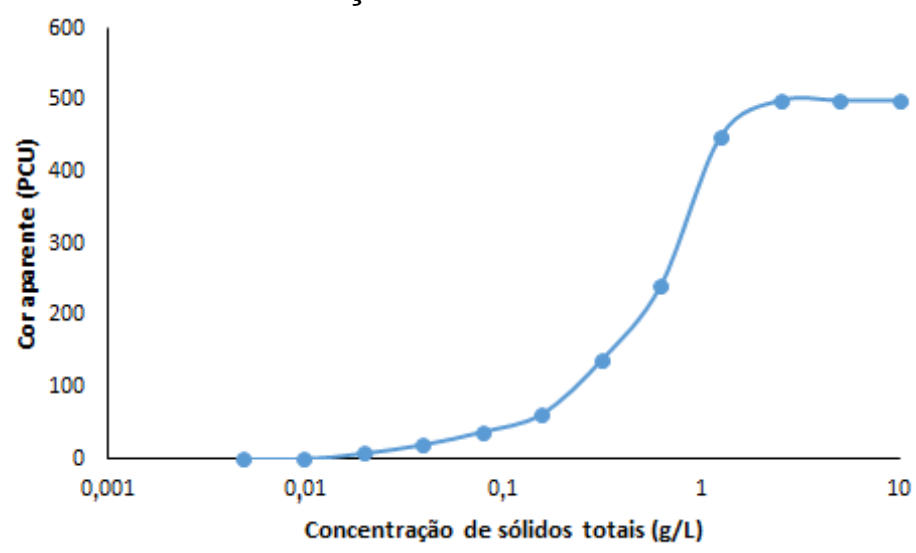

Fonte: Os autores. 


\section{Execução do experimento}

O objetivo do experimento é avaliar a capacidade de retenção de sedimentos do RSPA oriundos da primeira chuva. O sistema de alimentação e o RSPA foram montados com os materiais prescritos anteriormente, como se observa na Figura 6. A Figura 7, por sua vez, apresenta em destaque uma visão panorâmica da área próxima ao local de inserção dos sedimentos e de conexão do RSPA com o sistema de alimentação. Nota-se que o RSPA elaborado foi instalado no local pré-estabelecido no IFMG Campus Santa Luzia, conforme Figuras 6 e 7.

Com o sistema de alimentação montado, acoplado à entrada do RSPA e com os pontos de coleta e deposição de sedimentos verificados, inicia-se o experimento com a abertura do registro de alimentação de água.

A água inicia o escoamento pela tubulação de DN100, e quando passa pelo ponto de depósito do sedimento, conforme apresentado na Figura 6 (item a), insere-se $10 \mathrm{~g}$ da mistura dos sedimentos preparados. Com a água escoando pelo sistema, o RSPA segue enchendo até atingir o seu volume útil. Nesse instante, fez-se a coleta de uma amostra de $200 \mathrm{ml}$, pelo ponto de amostragem, conforme identificado na Figura 6 (item c). A partir da primeira coleta, realizaram-se outras quatro amostragens espaçadas a cada 30 segundos, contemplando um tempo total de aproximadamente três minutos de experimento.

A eficiência de retenção de sedimentos do RSPA foi determinada com base na quantidade de sólidos retidos no RSPA em relação à quantidade total de sólidos introduzidos no sistema de alimentação.
As amostras coletadas foram numeradas de 1 a 5 pela ordem de amostragem e encaminhadas para análise de cor aparente, para, posteriormente, obter-se a sua concentração de sólidos totais, com o auxílio da curva de calibração do colorímetro.

\section{RESULTADOS, ANÁLISE E DISCUSSÃO}

\section{Cálculo do volume do RSPA}

Utilizando-se a metodologia empregada por Mierzwa et al. (2007), foram obtidos $t=11,91 \mathrm{~s} \mathrm{e}$ $V=18,81 \mathrm{~L}$. Sendo assim, o volume do RSPA encontrado, considerando-se todos os parâmetros adotados e calculados de maneira regionalizada, é de aproximadamente 20 litros, muito inferior ao recomendado pela norma NBR 15527:2007, que prescreve o descarte dos primeiros $2 \mathrm{~mm}$ de chuva na falta de um projetista, o que conceberia um RSPA de 16L, para uma cobertura de $80 \mathrm{~m}^{2}$.

\section{Construção do RSPA e do sistema de alimentação}

A Figura 5 mostra o RSPA fabricado em escala real com tubos e conexões de PVC, conforme as dimensões apresentadas na Figura 8.

Com relação ao sistema de alimentação, a Figura 6, conforme já apresentado, mostra o esquema de montagem do sistema de alimentação para simulação da chuva de projeto. Observa-se a saída do ponto d'água e entrada do sistema à esquerda da figura, até a entrada do reservatório à direita.

Figura 6: Sistema experimental montado, (item a) dosagem de sedimento; (item b) RSPA; (item c) ponto de amostragem

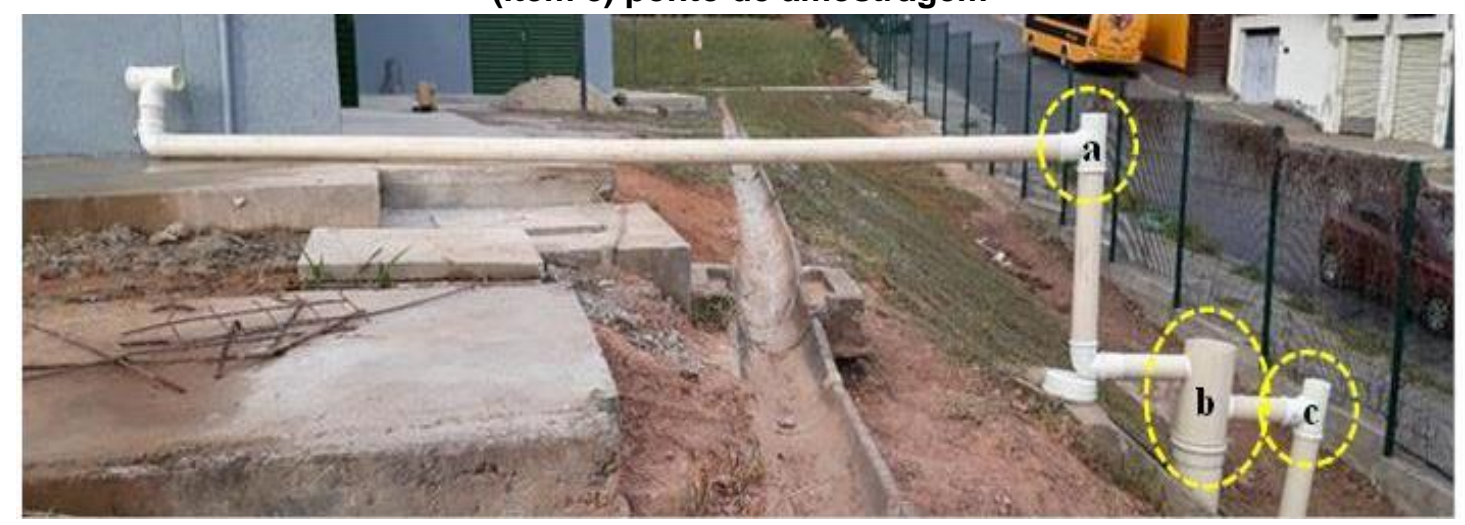

Fonte: Os autores. 
Figura 7: Vista superior RSPA e sistema de alimentação

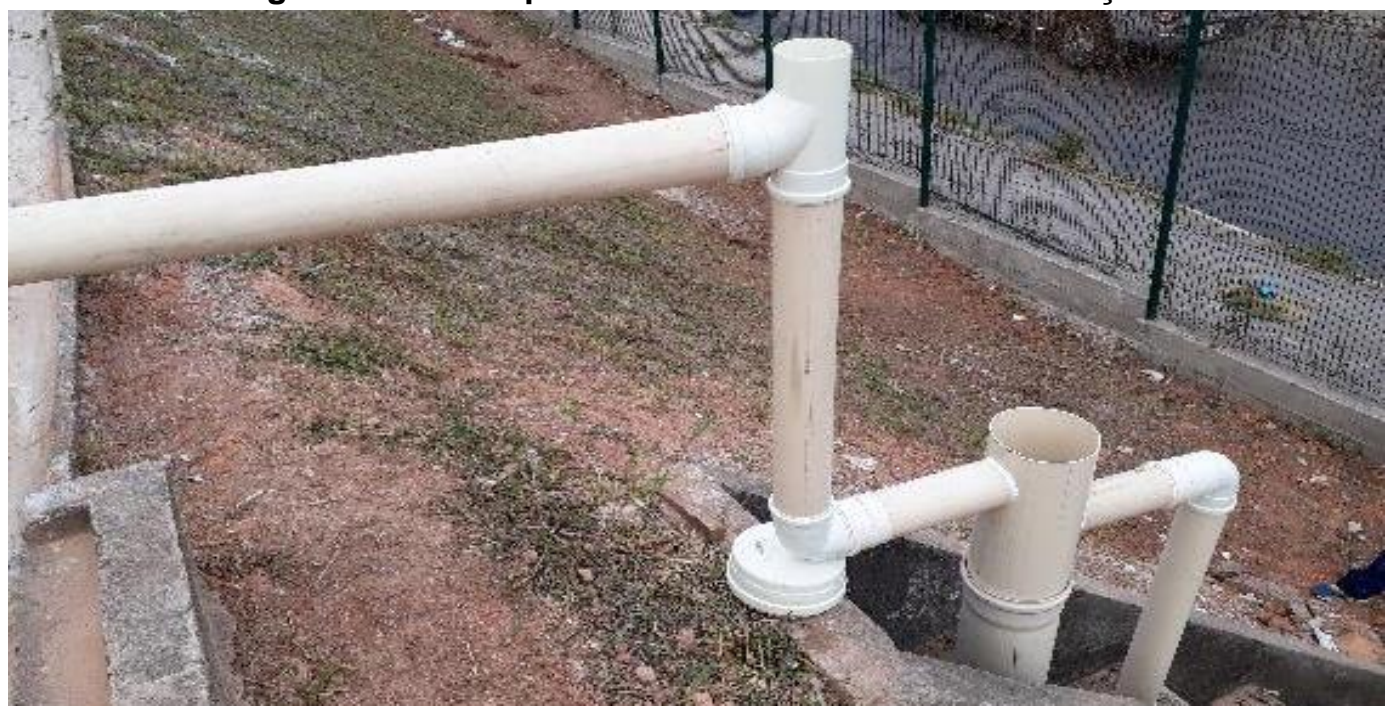

Fonte: Os autores.

Figura 8: RSPA construído em escala real com tubos e conexões de PVC

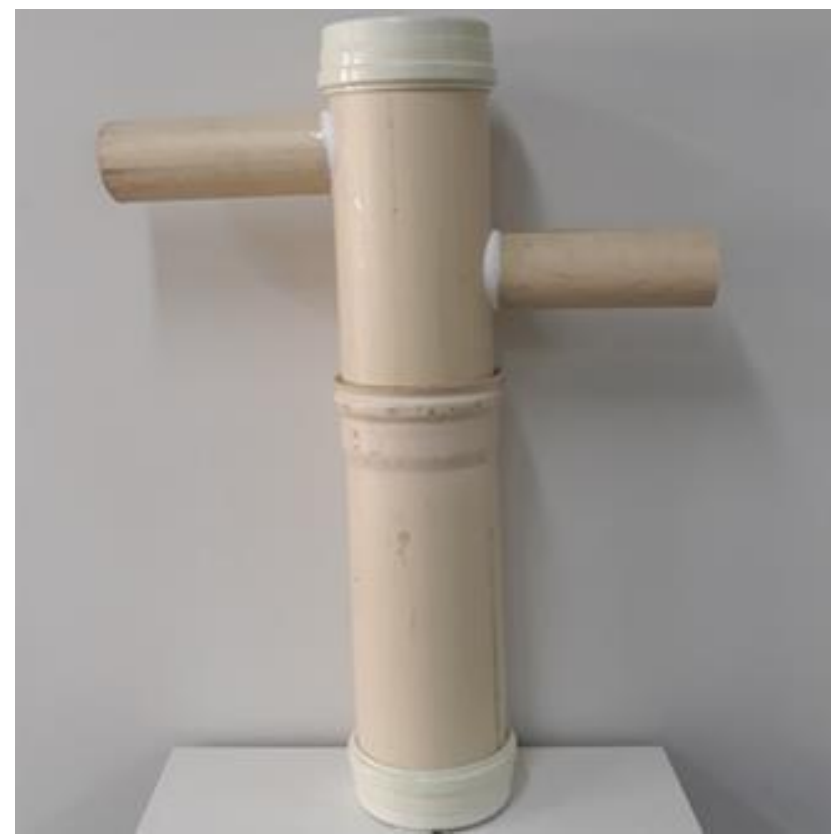

Fonte: Os autores.

\section{Execução do experimento}

Os resultados das medições de cor e da respectiva concentração de sólidos totais, obtidas por meio da curva de calibração para as amostras coletadas durante o ensaio experimental do RSPA, são apresentados na Tabela 2.

Tabela 2: Dados obtidos das amostragens

\begin{tabular}{l|c|c|c|c|c}
\hline $\mathrm{N}^{\circ}$ da amostra & 1 & 2 & 3 & 4 & 5 \\
\hline Cor aparente (PCU) & 40 & 65 & 5 & 0 & 0 \\
\hline Concentração de sólidos totais (g/l) & 0,085 & 0,170 & 0,016 & 0 & 0 \\
\hline
\end{tabular}

Fonte: Os autores. 
Com os resultados, observou-se uma concentração mais alta de sedimento nos primeiros minutos de ensaio e um ligeiro aumento de concentração entre as amostras 1 e 2 . Salienta-se que, no início do experimento, os sedimentos têm menos tempo para decantar, e observou-se visualmente a formação de turbulência nos primeiros instantes após 0 enchimento do reservatório, sendo as prováveis causas dos elevados valores iniciais da concentração de sólidos totais.

Da terceira amostra em diante, vê-se que a concentração de sólidos totais se reduz bruscamente até atingir o valor zero, o que se deve a uma estabilização do fluxo e diminuição da turbulência dentro RSPA, somado ao fato de que, nesses instantes, os sedimentos tiveram mais tempo para decantar. Sendo assim, nota-se que, após 2 minutos de realização dos testes, a saída de sólidos do RSPA já era desprezível, o que indica uma boa eficiência de retenção do sistema, já que a chuva de projeto possuía duração de 25min. Ressalta-se que, após 0 experimento, drenou-se o RSPA e notou-se grande quantidade de sólidos depositados no fundo.

Com relação à eficiência de remoção de sólidos, os resultados dos ensaios experimentais indicam uma eficiência de retenção de sedimentos no RSPA de cerca de $70 \%$, resultado expressivo evidenciando a elevada capacidade de remoção de sólidos do sistema avaliado. É importante destacar que a eficiência obtida é próxima do valor indicado em Novak, Giesen e Debusk (2014), os quais relataram eficiência de remoção de sólidos de $80 \%$ em sistemas RSPA, enquanto que Gardner, Baisden e Millar (2004) obtiveram taxas de retenção de sólidos de $65 \%$.

Além disso, Su (2007) obteve remoções entre $30 \%$ e $40 \%$, para sedimentos com características semelhantes ao utilizado no experimento em questão. A partir da comparação dos resultados obtidos com estudos semelhantes, nota-se que o RSPA proposto apresenta uma eficiência de remoção bastante promissora e que pode ainda ser aperfeiçoada em estudos futuros.

Os resultados reforçam a importância do RSPA na melhoria da qualidade da água enviada ao reservatório principal, conforme relatado em Tomaz (2012). Sendo assim, o RSPA projetado apresentou bons resultados de remoção de sedimentos da água coletada, mesmo tendo um volume oito vezes menor do que o recomendado pela norma NBR 15527:2007. Isso indica que o critério de dimensionamento adotado pela referida norma para o estudo de caso em questão resultaria em um superdimensionamento do RSPA, o que poderia aumentar o custo de implantação de um sistema de aproveitamento de água de chuva. Dessa forma, os critérios indicados na referida norma para dimensionamento do RSPA em sistemas similares deveriam ser alvo de atenção. Ressaltase que as discussões acima se aplicam apenas ao estudo de caso avaliado, o qual considerou uma edificação com área de captação de $80 \mathrm{~m}^{2}$. Logo, a generalização das afirmações acima não é recomendada, mas indica uma área potencial para estudos adicionais, considerando outras intensidades de chuva e áreas de captação, de modo a verificar a aplicabilidade das constatações aqui apresentadas a uma gama maior de situações.

\section{CONCLUSÕES}

O volume do RSPA encontrado, considerando-se todos os parâmetros adotados e calculados de maneira regionalizada, é de aproximadamente 20 litros, muito inferior ao recomendado pela norma NBR 15527:2007, que prescreve o descarte dos primeiros $2 \mathrm{~mm}$ de chuvas na falta de um projetista, o que conceberia um RSPA de 160 litros, para uma cobertura de $80 \mathrm{~m}^{2}$. Sendo assim, o estudo em questão demonstrou a eficiência da geometria do RSPA proposta quanto à retenção de sedimentos e indicou que para o estudo de caso em questão o procedimento de dimensionamento preconizado pelo NBR 15527:2007 resulta em valores de volume do referido reservatório mais elevados do que o necessário.

O material particulado utilizado no experimento apresentava diâmetros dos grãos inferiores a $0,106 \mathrm{~mm}$, em acordo com o material particulado encontrado na atmosfera observado pela literatura. Os dados obtidos com os ensaios e na construção da curva de calibração do colorímetro resultaram em dados coerentes e que não apresentavam discrepâncias, correspondendo a resultados fidedignos.

A geometria adotada para o RSPA se mostrou eficiente para a retenção dos sólidos utilizados. No início da realização do experimento, verificou-se um aumento da concentração de sólidos totais na saída do RSPA, com um pico máximo observado de $0,17 \mathrm{~g} / \mathrm{l}$, provavelmente, causado devido à turbulência durante o 
enchimento. Notou-se que, após 2 minutos de realização dos testes, a saída de sólidos do RSPA já era desprezível, o que indica uma boa eficiência de retenção do sistema já que a chuva de projeto possuía duração de $25 \mathrm{~min}$.

Sendo assim, recomenda-se em estudos futuros a avaliação de critérios a serem adotados na referida norma para dimensionamento de volumes do RSPA mais econômicos. Para isso, sugere-se a realização de estudos adicionais considerando outras intensidades de chuva e áreas de captação. Além disso, indica-se a avaliação experimental de outras geometrias para o RSPA, a fim de determinar qual seria a geometria ótima em termos de retenção de sedimentos. Propõe-se, também, a realização de ensaios de caracterização físico-química que envolva outros parâmetros de qualidade da água.

\section{AGRADECIMENTOS}

Agradecemos ao Instituto Federal de Minas Gerais por financiar o projeto de pesquisa que originou o estudo em questão, e ao campus Santa Luzia por propiciar estrutura física para a realização do experimento.

\section{REFERÊNCIAS}

ASSOCIAÇÃO BRASILEIRA DE NORMAS TÉCNICAS - ABNT. NBR 15527 - Água de chuva Aproveitamento de coberturas em áreas urbanas para fins não potáveis - Requisitos. Rio de Janeiro: ABNT, 2007, 12p.

ANDRADE NETO, C. O. Aproveitamento imediato da água de chuva. Gesta, Salvador, v.1, n.1, p. 73-86, 2013.

ASA - ARTICULAÇÃO NO SEMIÁRIDO BRASILEIRO. Disponível em: http://www.asabrasil.org.br Acesso em: 18 out. 2016.

BITTERMAN, P.; TATE, E.; VAN METER, K. J.; BASU, N. B. Water security and rainwater harvesting: a conceptual framework and candidate indicators. Applied Geography, Netherlands, v. 76, p. 75-84, 2016.

CARDOSO, M. P. Viabilidade do aproveitamento de água de chuva em zonas urbanas: estudo de caso no município de Belo Horizonte - MG. 2009. 192p. Dissertação (Mestrado) - DESA, UFMG, 2009.

COELHO, L. M. G.; ALVES, G. C. P.; VALLE, K. N. F. Avaliação de um reservatório de separação das primeiras águas via simulação hidráulica. In: SIMPÓSIO BRASILEIRO DE COLETA E MANEJO DE ÁGUAS DE CHUVA - SBCMAC, 11., 2018, João Pessoa. Anais... João Pessoa-PB: UFPB, 2018. 8p.

FERREIRA, V, H. C. Identificação das fontes de material particulado atmosférico fino e grosso no Distrito Federal empregando marcadores iônicos solúveis em água. 2016. 106p. Dissertação (Mestrado) - Universidade de Brasília, Brasília, 2016.

GARDNER, T.; BAISDEN, J.; MILLAR, G. Rainwater first flush devices - Are they effective? In: SUSTAINABLE WATER IN THE URBAN ENVIRONMENT, 2004, Brisbane. Proceedings... Brisbane, 2004.

LIMA, J. C. A.; CARVALHO, J. R. C.; FIGUEIRAS, M. L. Influência do descarte das primeiras águas de chuva sobre a qualidade da água encaminhada às cisternas. In: SIMPÓSIO BRASILEIRO DE COLETA E MANEJO DE ÁGUAS DE CHUVA - SBCMAC, 10., 2016, Belém. Anais... Belém-PA: UFPA, 2016.

MARTINSON, B.; THOMAS, T. Quantifying the firstflush phenomenon: effects of first-flush on water yield and quality. In: INTERNATIONAL RAINWATER CATCHMENT SYSTEMS CONFERENCE, 14., 2009, Tahun. Anais... Tahun: NAHRIM, 2009.

MEDEIROS, S. S.; SANTOS, D. B.; BRITO, L. T. L.; COHIM, E. et al. Captação manejo e uso de água de chuva. Campina Grande-PB: INSA, 2016. 460 p.

MELO, L. R. C.; ANDRADE NETO, C. O. Variação da qualidade da água de chuva em três pontos distintos da cidade de Natal-RN. In: CONGRESSO BRASILEIRO DE ENGENHARIA SANITÁRIA AMBIENTAL, 24., 2007, Belo Horizonte. Anais... Belo Horizonte-MG: ABES, 2007.

MIERZWA, J. C; HESPANHOL, I.; SILVA, M. C. C.; RODRIGUES, L. Águas pluviais: método de cálculo do reservatório e conceitos para um aproveitamento adequado. REGA, v. 4, n. 1, p. 29-37, jan. 2007.

NOTARO, V.; LIUZZO, L.. FRENI, G. Reliability analysis of rainwater harvesting systems in Southern Italy. Procedia Engineering, v. 162, p. 373-380, 2016.

NOVAK, C. A.; GIESEN, E. V.; DEBUSK, K. M. Designing rainwater harvesting systems: integrating rainwater into building systems. New York: Ed. Wiley, 2014. 312p.

OHNUMA, A. A.; SILVA, L. P.; PICCOLI, R.; PEIXE, C. Qualidade de águas pluviais em regime de first flush. In: ENCONTRO NACIONAL DE ÁGUAS URBANAS, 10., 2014, São Paulo. Anais... São Paulo-SP: ABRH, 
2014. $4 p$

PICCOLI, R. A. Análise físico-química da qualidade das águas pluviais: estudo de caso - Instituto de Aplicação Fernando Rodrigues da Silveira, Rio Comprido - RJ. 2015. 137p. Dissertação (Mestrado) Universidade do Estado do Rio de Janeiro, Rio de Janeiro, 2015.
SU, Y. Storm water runoff first flush modeling and treatment with a hydrodynamic device. Ohio: RCETOU, 2007. 233p.

TOMAZ, P. Aproveitamento de água de chuva para áreas urbanas e fins não potáveis. São Paulo: Navegar Editora, 2012. 279p. 